\title{
Revalidation of morphological characteristics and multiplex PCR for the identification of three congener invasive Liriomyza species (Diptera: Agromyzidae) in China
}

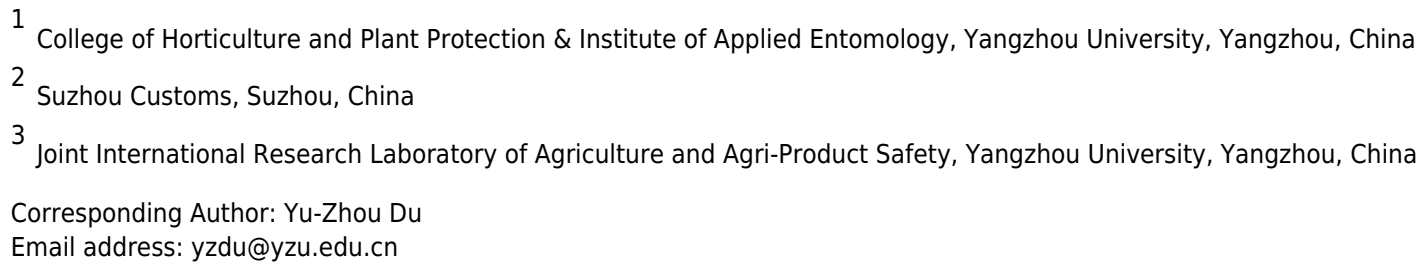

Due to varietal differences, diminutive size, and similar morphological characters, it is difficult to classify and identify Liriomyza spp., a genus comprised of economicallyimportant, highly-polyphagous insect pests. In this study, we reconfirmed the morphological characteristics of three closely-related invasive leafminers, $L$. trifolii, $L$. sativae, and L. huidobrensis. Morphological results showed that characteristics imparted by the male genitalia were the most reliable morphological features for identification. The colors exhibited by vertical setae were variable among species, and the ratio of the length of the ultimate section of vein $\mathrm{CuA}_{1}$ divided by penultimate section also varied within species. Although the patterns of abdominal tergites were diverse among Liriomyza spp.,

L. trifolii exhibited a unique pattern with a yellow patch at the $5^{\text {th }}$ black visible tergite; this pattern can be profiled as a prominent characteristic for morphological identification. In order to identify the three Liriomyza spp. quickly and accurately, we developed an improved molecular identification method using multiplex PCR based on the gene encoding mitochondrial cytochrome oxidase I (COI); this method enabled direct identification based on the size of amplified products. The results of this study provide a valuable reference for the identification of Liriomyza spp., which will ultimately improve our ability to control individual species. 
1 Revalidation of morphological characteristics and multiplex PCR for the

2 identification of three congener invasive Liriomyza species (Diptera:

3 Agromyzidae) in China

4

5 Ya-Wen Chang ${ }^{1}$, Jing-Yun Chen ${ }^{1,2}$, Si-Zhu Zheng ${ }^{2}$, Yuan Gao $^{2}$, Yun-Fang Chen $^{2}$, Yan-Feng

$6 \quad$ Deng ${ }^{2}, \mathrm{Yu}-\mathrm{Zhou} \mathrm{Du}^{1,3 *}$

7

$8{ }^{1}$ College of Horticulture and Plant Protection \& Institute of Applied Entomology, Yangzhou

9 University, Yangzhou, China

$11{ }^{2}$ Suzhou Customs, Suzhou, China

12

3 Joint International Research Laboratory of Agriculture and Agri-Product Safety, Yangzhou

14 University, Yangzhou, China

*Corresponding author: Yu-Zhou Du, e-mail: yzdu@yzu.edu.cn. 


\section{ABSTRACT}

Due to varietal differences, diminutive size, and similar morphological characters, it is difficult to classify and identify Liriomyza spp., a genus comprised of economically-important, highlypolyphagous insect pests. In this study, we reconfirmed the morphological characteristics of three closely-related invasive leafminers, L. trifolii, L. sativae, and L. huidobrensis.

Morphological results showed that characteristics imparted by the male genitalia were the most reliable morphological features for identification. The colors exhibited by vertical setae were variable among species, and the ratio of the length of the ultimate section of vein $\mathrm{CuA}_{1}$ divided by penultimate section also varied within species. Although the patterns of abdominal tergites were diverse among Liriomyza spp., L. trifolii exhibited a unique pattern with a yellow patch at the $5^{\text {th }}$ black visible tergite; this pattern can be profiled as a prominent characteristic for morphological identification. In order to identify the three Liriomyza spp. quickly and accurately, we developed an improved molecular identification method using multiplex PCR based on the gene encoding mitochondrial cytochrome oxidase I (COI); this method enabled direct identification based on the size of amplified products. The results of this study provide a valuable reference for the identification of Liriomyza spp., which will ultimately improve our ability to control individual species.

Keywords: Liriomyza, morphological characteristics, abdominal tergites, multiplex PCR, species identification, $C O I$ 
40 INTRODUCTION

41 Leafminer flies (Diptera: Agromyzidae), especially Liriomyza trifolii, L. sativae and $L$. 42 huidobrensis, are invasive insect pests in many countries. They are polyphagous, economicallysignificant pests that cause severe damage to many ornamental and vegetable crops worldwide

44 (Spencer, 1973, 1990; Reitz et al., 1999). Both larvae and adults cause serious damage to crops

on leaves can reduce photosynthetic capacity, and leaf mining activity can cause premature leaf drop resulting in reduced yields (Johnson et al., 1983; Chandler \& Gilstrap, 1987). Moreover, indirect damage occurs when adults pierce leaves for feeding and oviposition, thus increasing plant susceptibility to disease (Zitter \& Tsai, 1977; Motteoni \& Broadbent, 1988). The rapid life cycle and high growth rate of Liriomyza spp. can lead to serious crop losses. Accurate identification of Liriomyza is important for implementing effective control strategies, because insecticide resistance and tolerance to environmental stress varies among species (Chang et al., 2017; Gao et al., 2017). 1995; Lei et al., 1996; Chen, 1999; Scheffer et al., 2001), and adult males can only be identified

al., 2000; Scheffer et al., 2001).

60

Since morphological identification of female adults, larvae and pupae of Liriomyza species is 
complex and difficult, molecular methods of identification are required. Immature developmental stages are the most common forms intercepted at ports of entry, therefore, it is important to identify these interceptions accurately and rapidly. With the development of mitochondrial and other molecular markers (Carapelli et al., 2018; Chen et al., 2019), several molecular methods have been developed to identify Liriomyza species (Menken \& Ulenberg, 1983; Zehnder et al., 1983; Oudman et al., 1995; Chiu et al., 2000; Morgan et al., 2000). Multiplex PCR is a cost-effective, rapid, accurate method where identification can be determined by PCR product size with speciesspecific primers (Nakamura et al., 2013).

In this study, we re-verified morphological characteristics of three leafminers, L. trifolii, $L$. sativae and L. huidobrensis. A new morphological characteristic for detection of L. trifolii was investigated, and an improved molecular method for identification was developed based on multiplex PCR. This study provides approaches that can be deployed for identification of Liriomyza species, which will ultimately help future control efforts.

MATERIALS AND METHODS

\section{Insects}

The three species of Liriomyza spp. were collected from areas where leafminers occur in China. In this study, 263 individuals of three species were selected for further data analysis (Table S1).

These were collected at the larval stage, tagged with relevant information and transported to the laboratory for pupation and emergence as adults. After preliminary morphological identification, adults were labeled, immersed in $70 \%$ ethanol and stored at $-20^{\circ} \mathrm{C}$. After dissecting and 
82

photographing the samples, the remaining tissues were stored in 100\% ethanol for DNA extraction and molecular analysis.

\section{Morphological identification}

Samples were examined with a stereomicroscope (Zeiss Stemi 2000c) and photographed with a wide depth of field (Zeiss Smartzoom 5). Male genitalia and wings were dissected, and slides were prepared and photographed with the Axio imager A2 (Zeiss, Germany).

Differences in the ratios of ultimate section lengths of vein $\mathrm{CuA}_{1}$ among different Liriomyza species were determined by one-way analysis of variance (ANOVA), followed by Tukey's multiple comparisons. All statistical analyses were performed using SPSS v. 16.0 (SPSS, Chicago, IL, USA), and statistical significance was determined when $P<0.05$.

\section{Molecular identification and primer selection for multiplex PCR}

Genomic DNA of Liriomyza species was extracted using the AxyPrep ${ }^{\mathrm{TM}}$ Multisource Genomic DNA Kit (Axygen, USA). A partial sequence of the mitochondrial cytochrome oxidase I (COI) gene was amplified with common primers F, 5'-CAACATTTATTTTGATTTTTTGG-3' and R, 5'- TCCAATGCACTAATCTGCCATATTA-3' (Simon et al., 1994; Yang et al., 2010) using protocols described by Chen et al. (2019), to molecular cross-checking and verification all of Liriomyza species in this study using sequencing, accession number can be found in Table S1. For multiplex PCR, full-length COI genes of three Liriomyza species were downloaded from NCBI (https://www.ncbi.nlm.nih.gov/) and aligned using Clustal X. To develop a rapid 
103

104

105

identification method, three species-specific primers and a common reverse primer were mixed to amplify DNA from different Liriomyza species. The PCR conditions were as follows: denaturation at $94{ }^{\circ} \mathrm{C}$ for $3 \mathrm{~min} ; 35$ cycles at $94{ }^{\circ} \mathrm{C}$ for $1 \mathrm{~min}, 58^{\circ} \mathrm{C}$ for 1 min and $72{ }^{\circ} \mathrm{C}$ for 1 min; followed by extension at $72{ }^{\circ} \mathrm{C}$ for $10 \mathrm{~min}$. PCR was conducted in a $25 \mu \mathrm{L}$ reaction volume containing $2 \mu \mathrm{L}(100 \mathrm{ng})$ of DNA template, $1 \mu \mathrm{L}(10 \mu \mathrm{M})$ of each primer, $12.5 \mu \mathrm{L}$ of $2 \times$ Taq

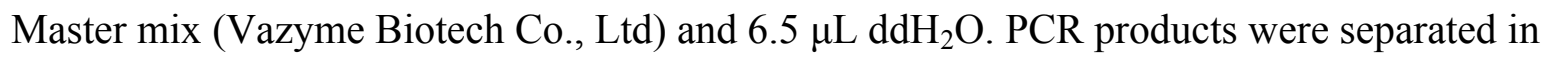
$1.0 \%$ agarose gels, and primers that amplified only one specific band for each species are shown in Table 1.

\section{RESULTS}

\section{Morphological identification}

The distiphallus, which is part of the male genitalia, is a very small, fragile structure enclosed by membranes located at the terminus of the aedeagus. For L. trifolii, the morphological

characteristics of the distiphallus include one distal bulb with marked constriction between lower and upper halves in dorsoventral view; the bulb is lightly sclerotized with a long basal stem (Fig. 1A). For L. sativae, the distiphallus is characterized by one distal bulb with a slight constriction between upper and lower halves in the dorsoventral view; the bulb is more intensely sclerotized with a shorter basal stem (Fig. 1B). For L. huidobrensis, the distiphallus contains two distal bulbs; these meet at rims that extend in an anteroventral orientation (Fig. 1C). With respect to vertical setae, L. trifolii exhibits inner and outer vertical setae on a yellow background; whereas vertical setae are present on a black background for L. huidobrensis. In $L$. 
124

125

126

127

128

129

130

131

132

133

134

135

136

137

138

139

140

141

142

143

144

sativae, outer and inner vertical setae are presented on black and yellow backgrounds, respectively (Spencer, 1973). In this study, only 86.1\% (192/223) of L. trifolii had yellow inner and outer vertical setae; $9.9 \%(22 / 223)$ had yellow inner vertical setae and undetermined color for outer setae, and 4.0\% (9/223) had yellow inner and black outer vertical setae (Table 2; Figs. 2A-C). For L. sativae, $17.6 \%(6 / 34)$ had black inner and outer vertical setae, $58.8 \%(20 / 34)$ had yellow inner and black outer vertical setae, and 23.5\% (8/34) had outer black setae with an undetermined color for inner vertical setae (Table 2; Fig. 2D-F). For L. huidobrensis, 100\% (6/6) exhibited black inner and outer vertical setae (Table 2; Fig. 2G-I). These results show that characteristics of vertical setae are not reliable for identifying Liriomyza species.

Wing pattern ratios were calculated as the length of the ultimate section of vein $\mathrm{CuA}_{1}$ divided by the penultimate section ('a' and 'b', see Fig. 3A-C). In this study, 'a' was $2.70 \pm 0.31$ times the length of ' $b$ ' in $L$. trifolii, and ' $a$ ' was $2.72 \pm 0.37$ times the length of ' $b$ ' in L. sativae.

For L. huidobrensis, 'a' was $2.20 \pm 0.24$ times the length of 'b' $\left(F_{2,237}=7.345, P<0.05\right)$ (Fig. 4). Although the ratio of $L$. huidobrensis was significantly different from the other two species $(P<$ $0.05)$, there was no significant difference between $L$. trifolii and $L$. sativae $(P=0.907)$. Many $L$. trifolii individuals exhibited truncated or missing dm-cu cross veins. Furthermore, we noted inconsistency between left and right forewing patterns within individual samples (Fig. 3A, with dashed lines).

In L. trifolii, the $2^{\text {nd }}-5^{\text {th }}$ visible tergites were generally divided by a yellow medial furrow in male adults; furthermore, there was a yellow patch at the $5^{\text {th }}$ black visible tergite that can distinguish L. trifolii from other Liriomyza species (Figure 5A-C). In L. sativae and $L$. 
145 huidobrensis, only the second visible tergite is divided by a yellow medial furrow and no yellow

146 patch is evident on the $5^{\text {th }}$ tergite (Fig. 5D-I).

147

148 Molecular detection of Liriomyza spp.

149 Candidate primers for species-specific detection of Liriomyza were based on the alignment of

150262 (L. sativae), 612 (L. trifolii), and 959 (L. huidobrensis) COI sequences. We designed one

151 reverse primer, $1181 \mathrm{R}$, that was common to all three Liriomyza species. The position of forward

152 primers was selected to produce $<1000 \mathrm{bp}$ amplicons when paired with the reverse primer with at

153 least $300 \mathrm{bp}$ nucleotides between species. In addition, sites were selected where the number of

154 differential nucleotides was $>2$ bp to increase the specificity of the primers (Fig. 6).

155 The three Liriomyza species could be differentiated by specific PCR products in $1.0 \%$

agarose gels, and the resulting PCR products were 569, 919, and 222 bp for L. trifolii, L. sativae

and L. huidobrensis, respectively (Fig. 7A). The validity of multiplex PCR for identification was

further confirmed by using the system with different developmental stages; the approach worked

equally well for larvae, pupae and adults of the three Liriomyza species (Fig. 7B). Populations

from different geographical regions were also obtained to evaluate the reliability of species-

specific primers. The results obtained by multiplex PCR (Fig. S1) and subsequent sequence

analysis of $C O I$ (Fig. S2) showed that geography did not impact the reliability of primers.

\section{DISCUSSION}

The morphological characteristics used for Liriomyza identification have primarily followed 
166 Spencer's (1973) criteria. However, variability in life stages, emergence times and sample

167 preservation result in large differences in body color and markings, which can make current 168 morphological criteria unreliable for identification (Spencer, 1973; Kang et al., 1996; Shao, 169 2004).

170 Currently, the identification of Liriomyza spp. based on morphology is restricted to male

171

172

173

adults because there are no reliable features for species-level identification of female adults or immature developmental stages (EPPO, 2005). The identification of adults requires the examination of the male adult genitalia. In general, the distiphallus provides reliable detection of the three Liriomyza species and has considerable diagnostic value (Spencer, 1973; Shiao, 2004). However, differences in distiphalluses between species are subtle and dissection is difficult for nonprofessionals. Consequently, features of distiphallic structure should be cross-checked with other external morphological characteristics to ensure that identification is valid.

According to Spencer (1973), coloration of the vertical setae is an important external feature that can distinguish L. trifolii and L. sativae without dissection; however, this feature is unstable and lacks clear interspecific boundaries. Results of the current study show that reliance on coloration of vertical setae can result in misidentification of L. trifolii and L. sativae; thus, this feature should only be used as a supplement for identification. The ratio of the length of the ultimate section of vein $\mathrm{CuA}_{1}$ is unreliable since most ratio values overlapped among Liriomyza species. In this study, we also evaluated the patterns of abdominal tergites and discovered that the yellow patch at the $5^{\text {th }}$ black visible tergite of $L$. trifolii is a new, reliable morphological characteristic for identification. Similar findings were reported for abdominal color patterns for 
187 six Liriomyza species (Shiao, 2004).

188 Molecular methods for insect identification can be used with different developmental

189 stages, including immature stages where morphological features may be lacking. Furthermore,

190 molecular assays may facilitate identification of atypical or damaged samples. However, the

191 specificity of molecular assays may be limited because they were developed for a particular

purpose and evaluated against a restricted number of species (Nakamura et al., 2013). Multiplex

PCR assays were recently developed for identification of Liriomyza species (Miura et al., 2004;

Guan et al., 2006; Nakamura et al., 2013) and are based on amplification of a target gene region

using species-specific primer combinations. Multiplex PCR assays are easier and faster than

other molecular methods, such as RAPD-PCR, PCR-RFLP, DNA barcoding and real-time PCR

(Chiu et al., 2000; Morgan et al., 2000; Scheffer et al., 2001; Kox et al., 2005; Scheffer et al.,

2006; Blacket et al., 2015; Sooda et al., 2017); furthermore, multiplex PCR assays are more

sensitive than enzyme electrophoresis methods (Zehnder et al. 1983; Menken \& Ulenberg, 1983,

1986; Oudman et al., 1995). In general, the reliability and sensitivity of multiplex PCR

represents a great improvement in molecular identification protocols and will enable us to

manage invasive pests more effectively.

CONCLUSIONS

Invasive Liriomyza spp. comprise a group of insect pests that cause considerable economic loss and serious quarantine problems. In this study, morphological features were re-evaluated for $L$.

trifolii, L. sativae, and L. huidobrensis, and the discriminative ability of traditional 
208 morphological characteristics, such as male genitalia, abdominal color patterns, length of $\mathrm{Cu}_{1}$

209 and abdominal tergite patterns were reevaluated. Furthermore, we developed an improved

210 molecular identification method using multiplex PCR based on COI to identify the three

211 Liriomyza species quickly and accurately. This study provides valuable tools for the

212 identification of Liriomyza spp. using both morphological and molecular criteria. 


\section{REFERENCES}

215 Blacket M J, Rice A D, Semeraro L, Malipatil M B. 2015. DNA-based identifications reveal 216 multiple introductions of the vegetable leafminer Liriomyza sativae (Diptera, Agromyzidae) 217 into the Torres Strait Islands and Papua New Guinea. Bulletin Entomology Research 105: $218 \quad 533-544$. 
Agromyzidae). Journal of Economic Entomology 76: 1061-1063.

Kang L. 1996. Ecology and sustainable control of serpentine leafminers. Science Press, Beijing.

Kox L F F, Van-den Beld H E, Lindhout B I, De Goffau L J W. 2005. Identification of economically important Liriomyza species by PCR-RFLP analysis. EPPO Bulletin 35: 7985.

Lei Z R, Wang Y, Wen J Z. 1996. Identification of 11 kinds of leafminers on vegetables. Plant Protection 22: 40-43.

Menken S B J, Ulenberg S A. 1983. Diagnosis of the agromyzids Liriomyza bryoniae and $L$. trifolii by means of starch gel electrophoresis. Entomologia Experimentalis et Applicata 34: 205-208.

Minkenberg D P T M, Van Lenteren J C. 1986. The leafminers Liriomyza bryoniae and $L$. trifolii (Diptera, Agromizae), their parasites and host plants, A Review. Agricultural University Wageningen Paper 86: 1-50.

Miura K, Tagami Y, Ohtaishi M, Iwasaki A. 2004. Application of molecular techniques to distinguish Liriomyza trifolii from L. sativae on tomato cultivation in Japan. Journal of Economic Entomology 97: 964-969.

Morgan, D J W, Reitz, S R, Atkinson P W, Trumble J T. 2000. The resolution of Californian populations of Liriomyza huidobrensis and Liriomyza trifolii (Diptera, Agromyzidae) using PCR. Heredity 85: 53-61.

Motteoni J A, Broadbent A B. 1988. Wounds caused by Liriomyza trifolii (Diptera, Agromyzidae) as sites for infection of Chrysanthemum by Pseudomonas cichorii. Canadian Journal of Plant Pathology 10: 47-52.

Musgrave C A, Poe S L, Bennett D R. 1975. Leaf miner population estimation in polycultured vegetables. Proceedings of the Florida State Horticultural Society 88: 156-160.

Nakamura S, Masuda T, Mochizuki A, Konishi K, Tokumaru S, Ueno K, Yamaguchi T. 2013. Primer design for identifying economically important Liriomyza species (Diptera, Agromyzidae) by multiplex PCR. Molecular Ecology Resources 13: 96-102.

Oudman L, Aukema B, Menken S B J, Ulenberg S A. 1995. A procedure for identification of polyphagous Liriomyza species using enzyme electrophoresis. EPPO Bulletin 25: 349- 
272

273

274

275

276

277

278

279

280

281

282

283

284

285

286

287

288

289

290

291

292

293

294

295

296

297

298

299

300

355.

Reitz S R, Kund G S, Carson W G, Phillips P A, Trumble J T. 1999. Economics of reducing insecticide use on celery through low-input pest management strategies. Agriculture, Ecosystems and Environment 73: 185-197.

Scheffer S J, Lewis M L, Ravindra C J. 2006. DNA barcoding applied to invasive leafminers (Diptera, Agromyzidae) in the Philippines. Annals of the Entomological Society of America 99: 204-210.

Scheffer S J, Lewis M L. 2001. Two nuclear genes confirm mitochondrial evidence of cryptic species within Liriomyza huidobrensis. Annals of the Entomological Society of America 94: 648-653.

Scheffer S J, Wijesekara A, Visser D, Hallet R H. 2001. Polymerase chain reaction-restriction fragment-length polymorphism method to distinguish Liriomyza huidobrensis from L. langei (Diptera, Agromyzidae) applied to three recent leafminer invasions. Journal of Economic Entomology 94: 1177-1182.

Shiao S F. 2004. Morphological diagnosis of six Liriomyza species (Diptera, Agromyzidae) of quarantine importance in Taiwan. Applied Entomology and Zoology 39: 27-39.

Simon C, Frati F, Beckenbaeh A T, Crespi B, Liu H, Flook P. 1994. Evolution, weighting and Phylogeneties utility of mitoehondrial gene sequences and compilation of conserved polymerase chain reaction Primers. Annals of the Entomological Society of America 87: 651-701.

Sooda A, Gunawardana D, Li D M, Kumarasinghe L. 2017. Multiplex real-time PCR assay for the detection of three invasive leafminer species, Liriomyza huidobrensis, L. sativae and L. trifolii (Diptera, Agromyzidae). Austral Entomology 56: 153-159.

Spencer K A. 1973. Agromyzidae (Diptera) of economic importance 9, Series Entomologica. Bath, The Hague Publishers.

Spencer K A. 1990. Host Specialization in the World Agromyzidae (Diptera) 45, Series Entomologica, Kluwer, Dordrecht, The Netherlands.

Yang F, Cao J M, Du Y Z. 2010. Survey and molecular identification of Liriomyza trifolii in Jiangsu, China. Plant Protection 36: 108-111. 
301

302

303

304

305

306

307
Zehnder G W, Trumble J T, White W R, 1983. Discrimination of Liriomyza species (Diptera, Agromyzidae) using electrophoresis and scanning microscopy. Proceedings of the Entomological Society of Washington 85: 564-574.

Zitter T A, Tsai J H. 1977. Transmission of three potyviruses by the leafminer Liriomyza sativa (Diptera, Agromzidae). Plant Disease Reporter 61: 1052-1029. 


\section{FIGURE LEGENDS}

Figure 1. Lateral view of phalluses in three Liriomyza species. A, L. trifolii; B, L. sativae; C, L. huidobrensis.

Arrows indicate the distiphallus. Scale bar $=0.01 \mathrm{~mm}$.

Figure 2. Color characteristics of outer and inner vertical setae in three Liriomyza species. A-C, L. trifolii; D-F,

L. sativae; G-I, L. huidobrensis. Scale bar $=0.1 \mathrm{~mm}$. Yellow arrows indicate the position of outer vertical setae, and red arrows indicate the position of inner vertical setae.

Figure 3. Comparison of wing pattern ratios for three Liriomyza species. Ratios were calculated as the length of the ultimate section of vein $\mathrm{CuA}_{1}$ divided by the penultimate section ('a' and 'b' sections). A, L. trifolii; $\mathrm{B}, L$. sativae; C, L. huidobrensis. Scale bar $=0.1 \mathrm{~mm}$. The panel outlined with dashed lines represents an abnormal wing pattern in L. trifolii. Black arrows indicate the position of dm-cu cross veins.

Figure 4. Ratios showing lengths of the ultimate section of vein $\mathrm{CuA}_{1}$ divided by the penultimate section.

Differences among three Liriomyza species were determined by one-way analysis of variance (ANOVA), means median value. Abbreviations: Lt, L. trifolii; Ls, L. sativae, and Lh, L. huidobrensis.

Figure 5. Abdominal color patterns of three Liriomyza species. A-C, L. trifolii; D-F, L. sativae; G-I, L.

huidobrensis. Scale bar $=0.1 \mathrm{~mm}$. 
Figure 6. Alignment of COI sequences in Liriomyza spp. Sequences surrounded by rectangular boxes represent

primers used in this paper. Base substitutions are indicated by shading. Lt, L. trifolii; Ls, L. sativae; Lh, $L$.

huidobrensis.

Figure 7. Agarose gel electrophoresis of multiplex PCR products. (A) PCR products in three different

Liriomyza species. (B) PCR products from different developmental stages of the three Liriomyza species. Each

experiment contained three biological samples. Abbreviations: Lt, L. trifolii; Ls, L. sativae; Lh, L.

huidobrensis.

Figure S1. Agarose gel electrophoresis of multiplex PCR products from different geographical populations of

Liriomyza. Lt Lanes 1-10 indicate L. trifolii populations from: (1) Hengshui, (2) Hangzhou, (3) Dongguan, (4)

Zhangzhou, (5) Qionghai, (6), Nanning, (7) Changzhou, (8) Nanchang, (9), Huizhou, and (10) Huzhou. Ls lanes

1-2 indicate L. sativae populations from Shangqiu and Luoyang, respectively. Lh lanes 1-2 represent $L$.

huidobrensis populations from Kunming and the laboratory, respectively.

Figure S2. Alignment of COI sequences from different geographical population using species-specific primers.

The shaded nucleotides represent divergent sites. Nucleotides bounded by red and blue dashed rectangles

represent forward and reverse primers, respectively. Lt sequences represent the following L. trifolii populations:

Lt 1, Hengshui; Lt 2, Hangzhou; Lt 3, Dongguan; Lt 4, Zhangzhou; Lt 5, Qionghai; Lt 6, Nanning; Lt 7, 
350 from Shangqiu (Ls 1) and Luoyang (Ls 2). Lh sequences represent L. huidobrensis populations from Kunming

351 (Lh 1) and the laboratory ( $\operatorname{Lh} 2)$.

352 


\section{Table $\mathbf{1}$ (on next page)}

Table 1. Information of the primers designed in this study. 
2

3

Table 1. Primers used in this study.

\begin{tabular}{clccc}
\hline $\begin{array}{c}\text { Primer } \\
\text { name }\end{array}$ & \multicolumn{1}{c}{ Nucleotide sequence $\left(5^{\prime}-3^{\prime}\right)$} & $\begin{array}{c}\mathrm{Ta}(\mathrm{Tm}) \\
{ }^{\circ} \mathrm{C}\end{array}$ & $\begin{array}{c}\text { Product } \\
\text { size }(\mathrm{bp})\end{array}$ & $\begin{array}{c}\text { GenBank } \\
\text { number }\end{array}$ \\
\hline Lt612 & CAATTACAATACTATTAACAGACCG & $58(48.5)$ & 569 & MT919718 \\
Ls262 & AGCTCCAGACATAGCATTTCCTCG & $58(58.9)$ & 919 & MT919719 \\
Lh959 & TTCAGATGGCTTGCCACATTACACG & $58(59.9)$ & 222 & MT919720 \\
LR1181 & GAATAAATCCKGCTATAATTGCAAATAC & $58(50.9)$ & - & - \\
\hline
\end{tabular}

4 


\section{Table 2 (on next page)}

Table 2. The data of color characteristic of outer and inner vertical setae position in three Liriomyza species. 
1

2 Table 2. Color characteristics of outer and inner vertical setae in three Liriomyza species

\begin{tabular}{ccccccc}
\hline Species & $\begin{array}{c}\text { Vertical setae } \\
\text { position } \\
\text { (Inner/ Outer) }\end{array}$ & $\begin{array}{c}\text { Individual } \\
\text { phenotypes }\end{array}$ & $\begin{array}{c}\text { Vertical setae } \\
\text { position } \\
\text { (Inner/Outer) }\end{array}$ & $\begin{array}{c}\text { Individual } \\
\text { phenotypes }\end{array}$ & $\begin{array}{c}\text { Vertical setae } \\
\text { position } \\
\text { (Inner/ Outer) }\end{array}$ & $\begin{array}{c}\text { Individual } \\
\text { phenotypes }\end{array}$ \\
\hline \multirow{3}{*}{ L. trifolii } & $\mathrm{Y} / \mathrm{Y}$ & 192 & $\mathrm{~B} / \mathrm{Y}$ & 0 & $\mathrm{U} / \mathrm{Y}$ & 0 \\
& $\mathrm{Y} / \mathrm{B}$ & 9 & $\mathrm{~B} / \mathrm{B}$ & 0 & $\mathrm{U} / \mathrm{B}$ & 0 \\
\hline \multirow{3}{*}{ L. sativae } & $\mathrm{Y} / \mathrm{U}$ & 22 & $\mathrm{~B} / \mathrm{U}$ & 0 & $\mathrm{U} / \mathrm{U}$ & 0 \\
\hline \multirow{4}{*}{ L. huidobrensis } & $\mathrm{Y} / \mathrm{Y}$ & 0 & $\mathrm{~B} / \mathrm{Y}$ & 0 & $\mathrm{U} / \mathrm{Y}$ & 0 \\
& $\mathrm{Y} / \mathrm{B}$ & 20 & $\mathrm{~B} / \mathrm{B}$ & 6 & $\mathrm{U} / \mathrm{B}$ & 8 \\
& $\mathrm{Y} / \mathrm{U}$ & 0 & $\mathrm{~B} / \mathrm{U}$ & 0 & $\mathrm{U} / \mathrm{U}$ & 0 \\
\hline & $\mathrm{Y} / \mathrm{Y}$ & 0 & $\mathrm{~B} / \mathrm{Y}$ & 0 & $\mathrm{U} / \mathrm{Y}$ & 0 \\
& $\mathrm{Y} / \mathrm{B}$ & 0 & $\mathrm{~B} / \mathrm{B}$ & 6 & $\mathrm{U} / \mathrm{B}$ & 0 \\
\hline
\end{tabular}

3 Abbreviations: Y, yellow; B, black; and U, unclear.

4 
Figure 1

Photo plates of the phalluses of three Liriomyza species, lateral view.

A, L. trifolii; B, L. sativae; C, L. huidobrensis. Arrows indicate the distiphallus. Scale bar $=0.01$ $\mathrm{mm}$.

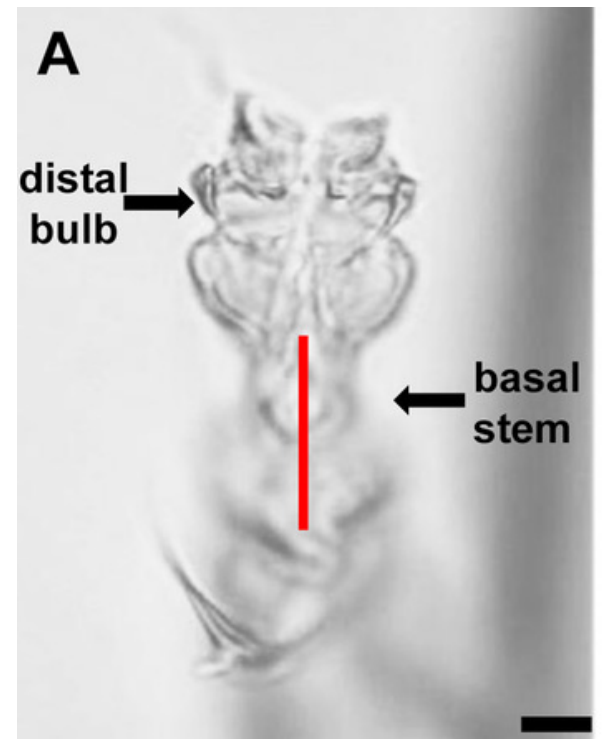

B

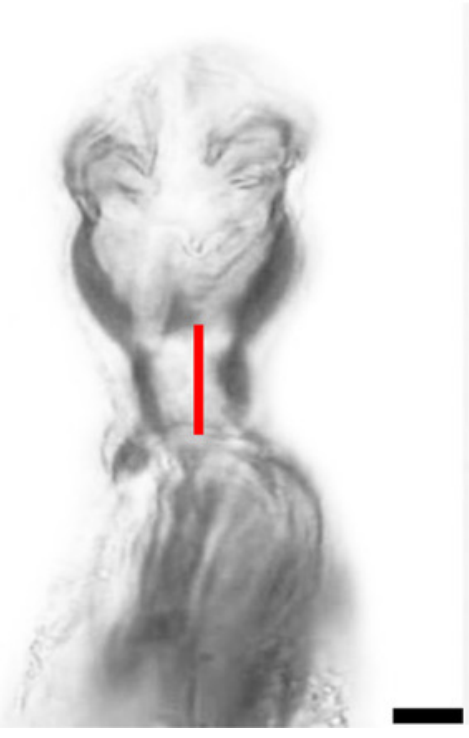

C

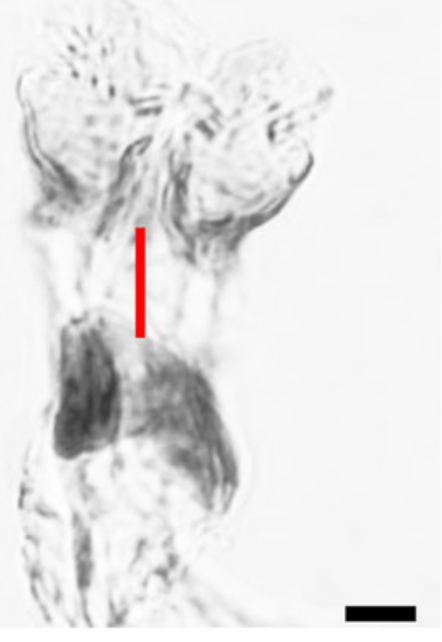




\section{Figure 2}

The color characteristic of outer and inner vertical setae position in three Liriomyza species.

A-C, L. trifolii; D-F, L. sativae; G-I, L. huidobrensis. Scale bar $=0.1 \mathrm{~mm}$. The yellow arrow indicated the position of outer vertical setae and the red arrow indicated the position of inner vertical setae.
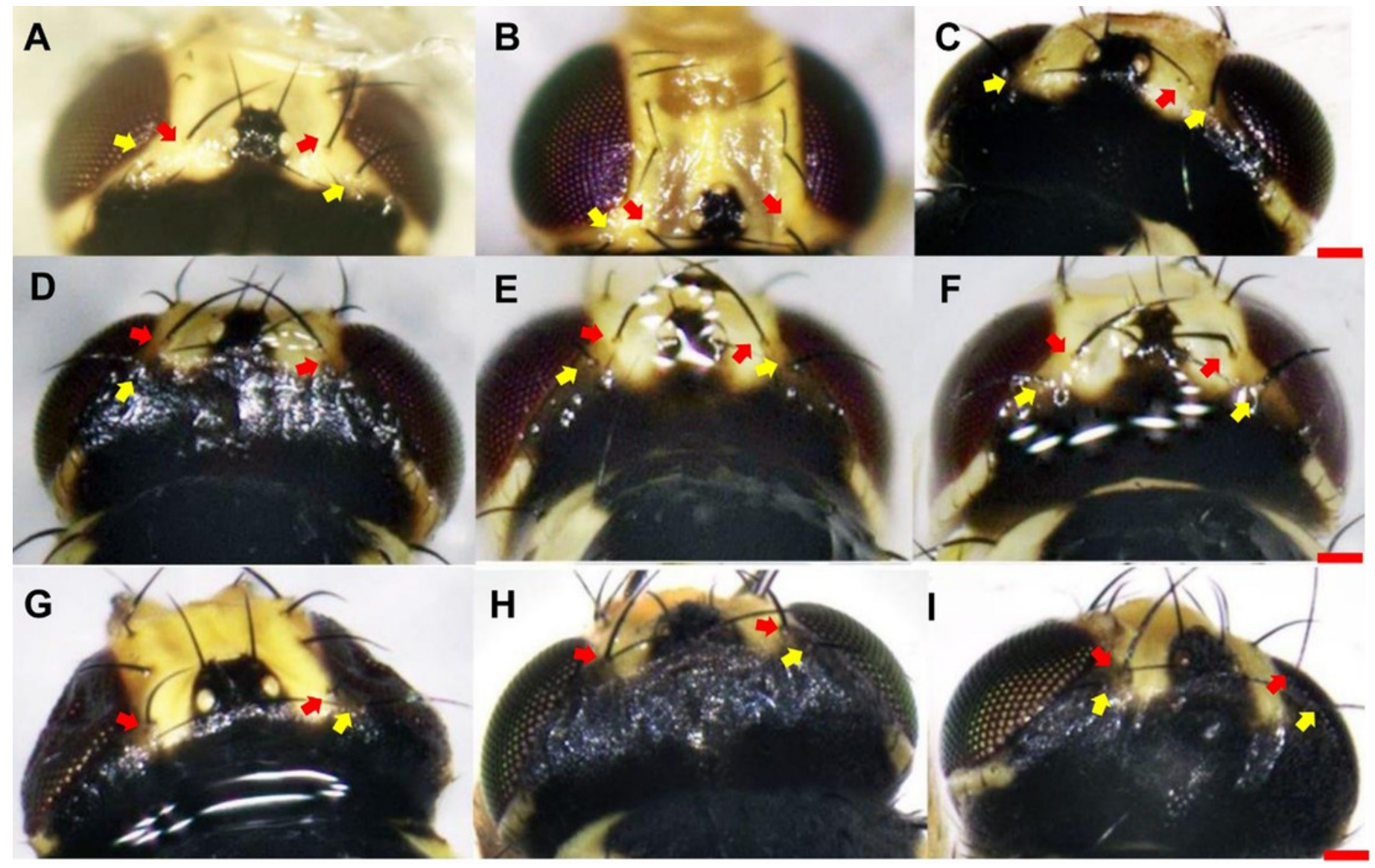
Figure 3

Comparison of the wing patterns of three Liriomyza species. The length of ultimate section of vein $\mathrm{CuA}_{1}$ divided by penultimate section ( $a$ and $b$ sections).

A, L. trifolii; B, L. sativae; C, L. huidobrensis. Scale bar $=0.1 \mathrm{~mm}$. Dot box represents abnormal wing pattern in L. trifolii. 

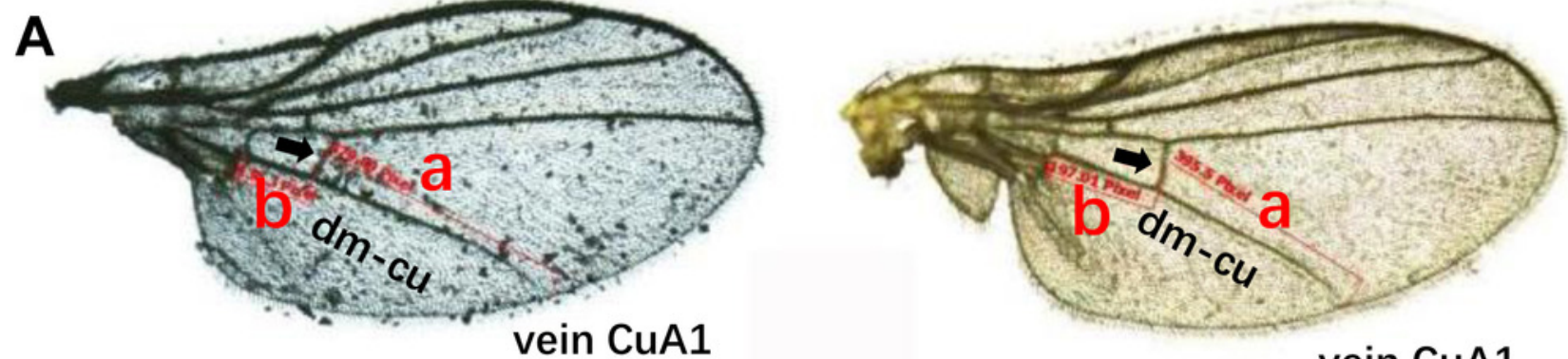

vein CuA1

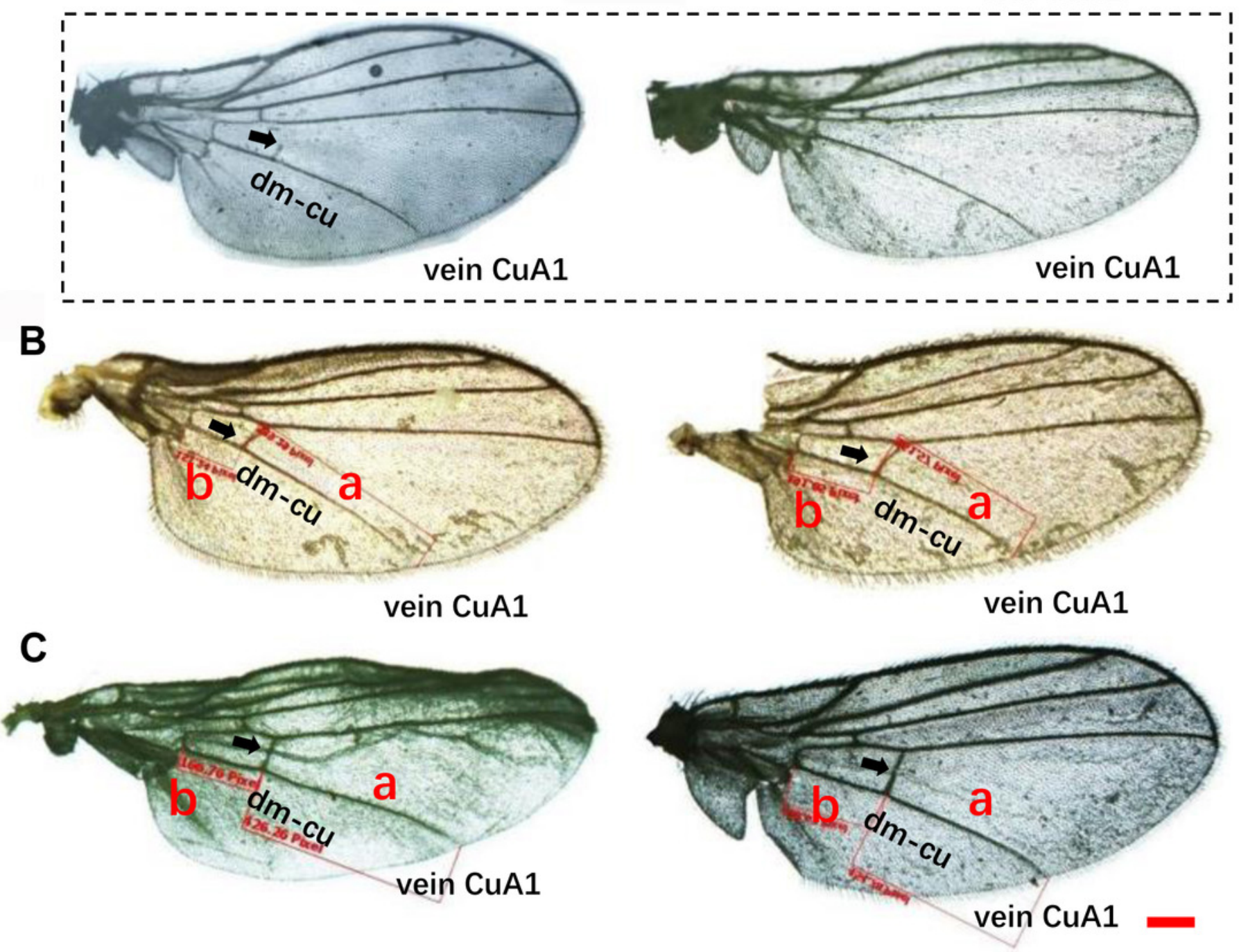


Figure 4

The ratio of the length of ultimate section of vein CuAl divided by penultimate section.

Differences in the ratio length of ultimate section of vein CuAl among different Liriomyza species were determined by one-way analysis of variance (ANOVA), followed by Tukey's multiple comparison $(P<0.05)$. The data in the figure is the average \pm standard deviation.

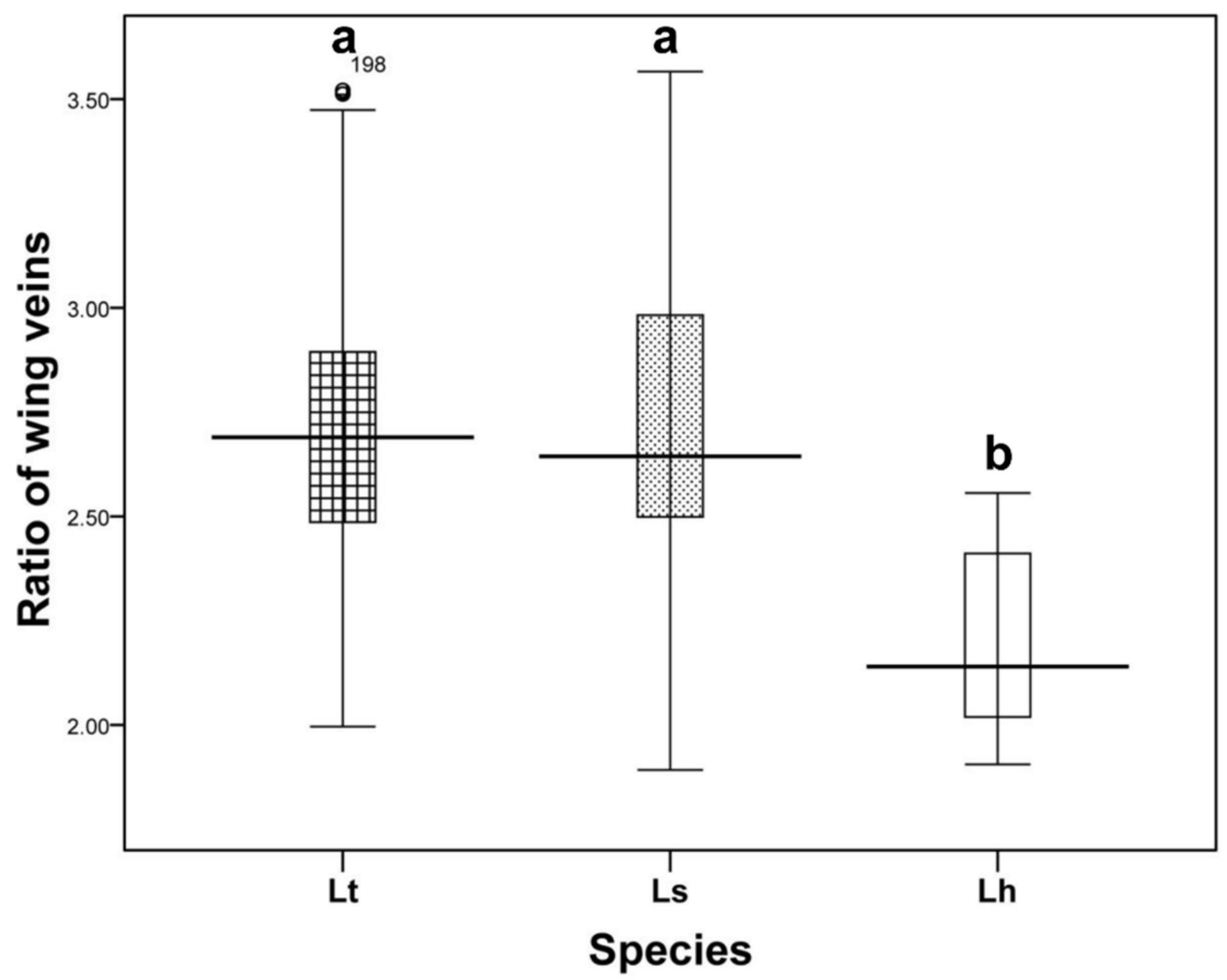


Figure 5

Diagrams of abdominal color patterns of three Liriomyza species.

A, L. trifolii; B, L. sativae; C, L. huidobrensis. Scale bar $=0.1 \mathrm{~mm}$.

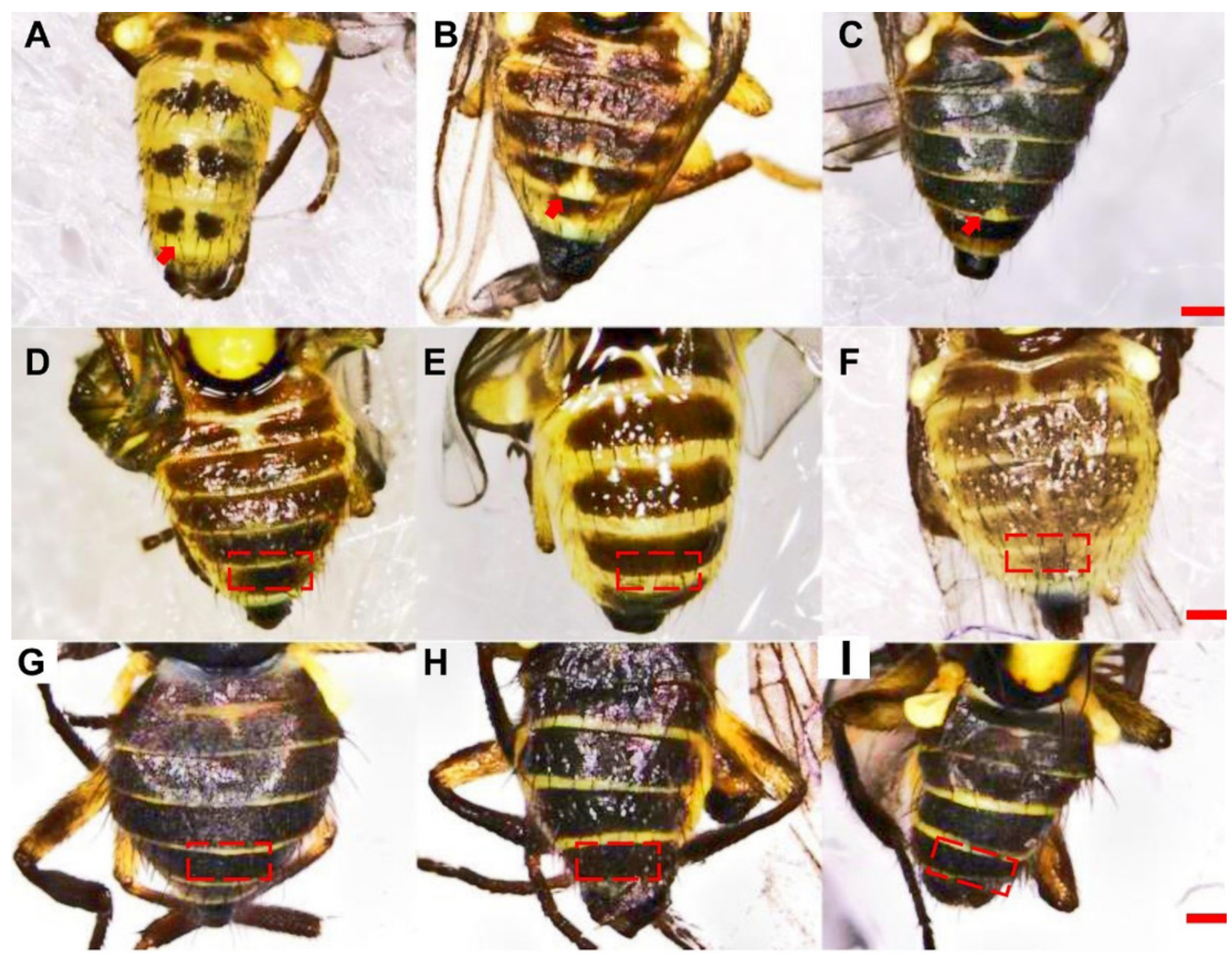


Figure 6

Alignment of $\mathrm{CO}$ sequences. Boxs indicate primer positions used in this paper.

Base substitutions are indicated by the shadow. Lt, L. trifolii; Ls, L. sativae; Lh, L. huidobrensis.

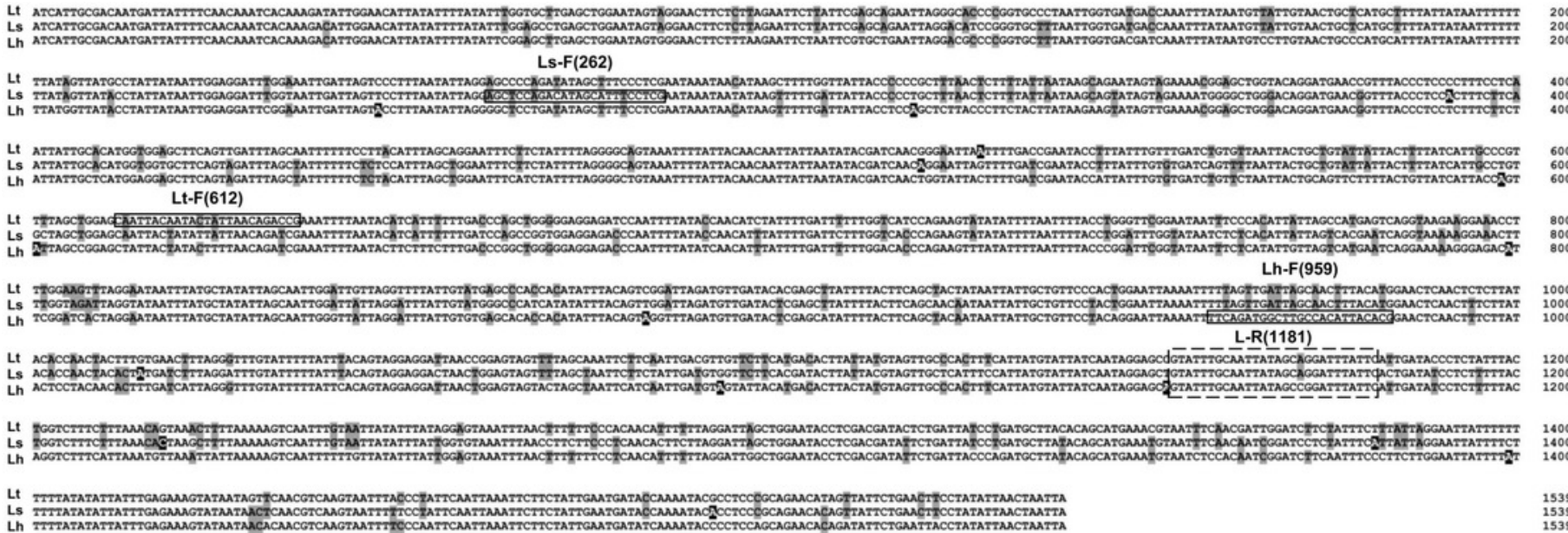




\section{Figure 7}

Agarose gel electrophoresis image of multiplex PCR products.

(A) DNA from different Liriomyza adults. (B) DNA from different developmental stages of $L$. trifolii. Each experiment has three biological repeats. Lt, L. trifolii; Ls, L. sativae; Lh, L. huidobrensis.

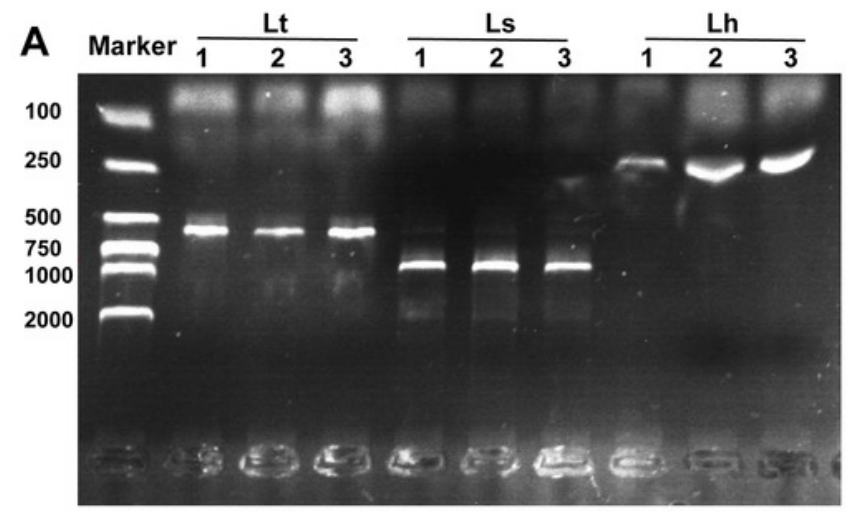

B
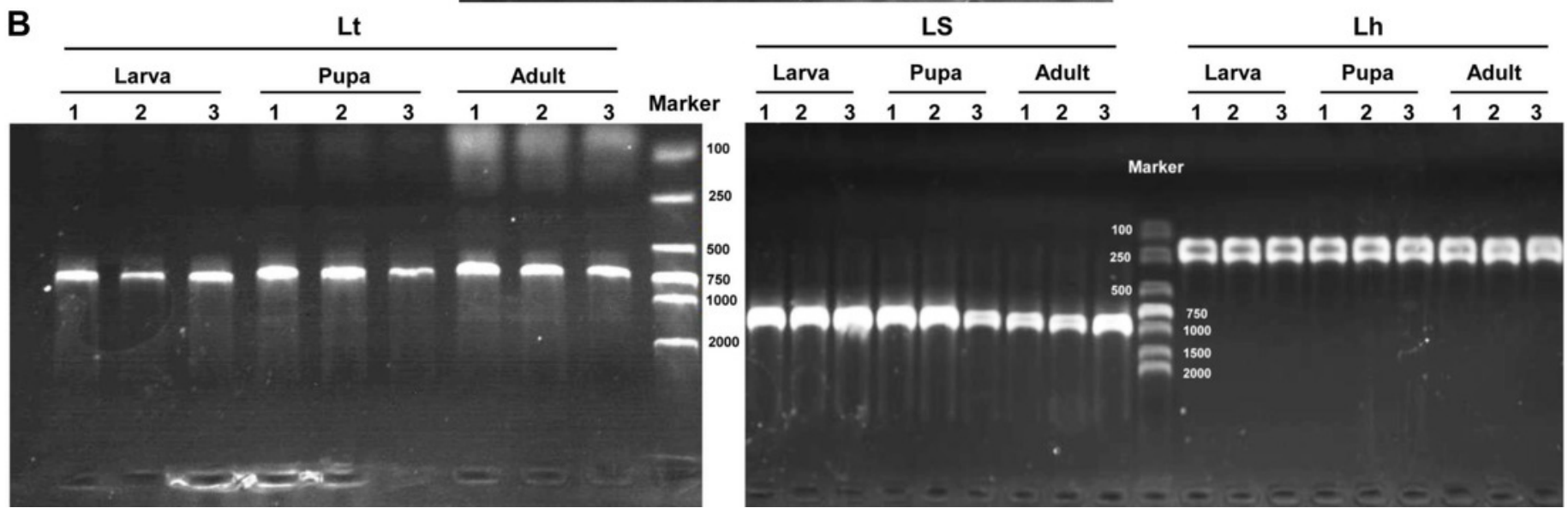

Marker

gagagagag

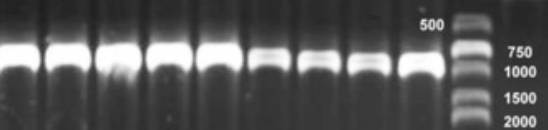

